\title{
INVERSION OF THE POISSON-HANKEL TRANSFORM
}

\author{
C. NASIM \\ Department of Mathematics \\ The University of Calgary \\ Calgary, Alberta \\ T2N IN4 CANADA
}

(Received July 15, 1980 and in revised form November 3, 1981)

ABSTRACT. The Poisson-Hankel transform is defined as an integral transform of the initial temperature function, with the kernel as the source solution of the generalized heat equation. In this paper a technique involving integral and differential operators has been used to effect the inversion of the Poisson-Hankel transform. KEY WOROS AND PHRASES. Heat equation, Poisson-Hankel transform, Gamma function, Bessel functions, whittaker function.

1980 MATHEMATICS SUBJECT CLASSIFICATION CODES. $44 A 15$.

1. INTRODUCTION.

The Poisson-Hankel transform has as its kernel the fundamental solution of the generalized heat equation. A special case of the Poisson-Hankel transform, called the reduced Poisson-Hankel transform has been studied in [1], where a differential operator of Laguerre-Polya class [2] has been used to effect its inversion. A general theory of these type of operators has been developed by Widder [2], but can not be applied to the more general Poisson-Hankel transform. Our object in this paper is to establish a procedure for the inversion of this transform in its general form. Our technique consists of applying an integral operator and a differential operator on the transform successively to retrieve the unknown function, cf [3]. The differential operator is of the Laguerre-Polya class.

We shall also deduce the inversions of the Weierstrass Hankel transform and the reduced Poisson-Hankel transform as special cases of our inversion algorithm. 
In the end we give an example to illustrate the result of the main theorem.

\section{DEFINITIONS AND PRELIMINARIES.}

The generalized heat equation is

$$
\frac{\partial^{2} u}{\partial x^{2}}+\frac{2 v}{x} \frac{\partial u}{\partial x}=\frac{\partial}{\partial t} u(x, t), \quad v \geq 0
$$

A $\mathrm{C}^{2}$ solution of (2.1) is called a generalized temperature. The fundamental solution of (2.1) is the function

$$
G(x ; t)=(2 t)^{-v-1 / 2} e^{-x^{2} / 4 t}
$$

We define the function associated with $G(x ; t)$ by

$$
G(x, y ; t)=2^{\nu-3 / 2} \Gamma\left(\nu+\frac{1}{2}\right) t^{-1}(x y)^{1 / 2-\nu} e^{-\frac{x^{2}+y^{2}}{4 t}} I_{\nu-1 / 2}\left(\frac{x y}{2 t}\right), v \geq 0,(2: 2)
$$

$I_{V}(z)$ being the Bessel function of imaginary argument and order $V$. The function $G(x, y ; t)$ is the source solution of the generalized heat equation (2.1). Note that $G(x, 0 ; t)=G(x ; t)$.

The Poisson-Hankel transform is defined by

$$
U(x, t)=\int_{0}^{\infty} G(x, y ; t) \phi(y) d \mu(y), \quad 0<t<\infty
$$

where $\quad d u(y)=\frac{2^{1 / 2-\nu}}{\Gamma\left(\nu+\frac{1}{2}\right)} y^{2 \nu} d y$.

The convergent Poisson-Hankel transform defines a generalized temperature $U(x, t)$

with initial temperature

$$
\mathrm{U}(\mathrm{x}, 0+)=\phi(\mathbf{x})
$$

Next, some operational considerations.

From the Euler product of the gamma function

$$
\Gamma(z)=\lim _{n \rightarrow \infty} \frac{1 \cdot 2 \cdot 3 \cdots(n-1)}{z(z+1) \cdots(z+n-1)} n^{z}
$$

one can easily show that

$$
\begin{aligned}
\frac{1}{\Gamma(\alpha-\beta z)} & =\lim _{n \rightarrow \infty} \frac{\beta^{n}}{(n-1) !} n^{-\alpha+\beta z} \prod_{k=1}^{n}\left(\frac{\alpha+k-1}{\beta}-z\right) \\
& =\lim _{n \rightarrow \infty} \frac{\beta^{n}}{(n-1) !} n^{-\alpha+\beta z} p_{n}(z),
\end{aligned}
$$


$p_{n}(z)$ being a polynomial in $z$ of order $n$. Now we define the operator

$$
\frac{1}{\Gamma\left(\nu+\frac{1}{2}-\frac{1}{2} \theta\right)}=\lim _{n \rightarrow \infty} \frac{n^{-v-1 / 2}}{(n-1) ! 2^{n}} n^{\theta / 2} p_{n}(\theta)
$$

where $\theta=-x \frac{d}{d x}$. Except for the factor $n^{\theta / 2}, \frac{1}{\Gamma\left(\nu+\frac{1}{2}-\frac{1}{2} \theta\right)}$ is the Euler differential operator. To obtain the intended interpretation of the operator $\mathrm{n}^{\theta / 2}$, we write

$$
\begin{aligned}
\mathrm{n}^{\theta / 2}=\mathrm{e}^{\theta \ln \mathrm{n} / 2} & =\lim _{\mathrm{N} \rightarrow \infty} \sum_{k=0}^{N}\left(\frac{\ln \mathrm{n}}{2}\right)^{k} \frac{1}{k !} \theta^{k} \\
& =\lim _{N \rightarrow \infty} \mathrm{q}_{N}(\theta)
\end{aligned}
$$

$q_{N}$, a polynomial in $\theta$ of degree $N$. To see the effect of $n^{\theta / 2}$ on a function $x^{\alpha}$, where $\alpha$ is a constant, first note that

$$
\theta^{n}\left[x^{\alpha}\right]=(-\alpha)^{n} x^{\alpha}
$$

and hence

a polynomial of degree $n$. Now,

$$
p_{n}(\theta)\left[x^{\alpha}\right]=p_{n}(-\alpha) x^{\alpha} \text {, where } p_{n} \text { is }
$$

$$
\begin{aligned}
n^{\theta / 2}\left[x^{\alpha}\right] & =\lim _{N \rightarrow \infty} q_{N}(\theta)\left[x^{\alpha}\right] \\
& =\lim _{N \rightarrow \infty} q_{N}(-\alpha) x^{\alpha} \\
& =n^{-\alpha / 2} x^{\alpha} .
\end{aligned}
$$

With this understanding, one can readily see that

$$
\frac{1}{\Gamma\left(\nu+\frac{1}{2}-\frac{1}{2} \theta\right)}\left[x^{\alpha}\right]=\frac{1}{\Gamma\left(\nu+\frac{1}{2}+\frac{1}{2} \alpha\right)} x^{\alpha}
$$

Thus, $\frac{1}{\Gamma\left(\nu+\frac{1}{2}-\frac{1}{2} \theta\right)}$ will be called a linear differential operator of infinite order and the effect of this operator on a function $x^{\alpha}$ is to reproduce it with a constant factor. This operator is of Laguerre-Pólya class and further properties of the operator of this class are well known, cf [5].

Next we give two applications of this operator for future reference. First, 


$$
\begin{aligned}
\frac{1}{\Gamma\left(\nu+\frac{1}{2}-\frac{1}{2} \theta\right)} \mathrm{e}^{-\mathrm{a}^{2} \mathrm{x}^{2}} & =\frac{1}{\Gamma\left(\nu+\frac{1}{2}-\frac{1}{2} \theta\right)} \sum_{\mathrm{k}=0}^{\infty} \frac{\left(-\alpha^{2} \mathrm{x}^{2}\right)^{\mathrm{k}}}{\mathrm{k} !} \\
& =\sum_{\mathrm{k}=0}^{\infty} \frac{\left(-\alpha^{2}\right)^{\mathrm{k}}}{\mathrm{k} !} \frac{1}{\Gamma\left(\nu+\frac{1}{2}-\frac{1}{2} \theta\right)}\left[\mathrm{x}^{2 \mathrm{k}}\right] \\
& =\sum_{\mathrm{k}=0}^{\infty} \frac{\left(-\alpha^{2}\right)^{\mathrm{k}}}{\mathrm{k} !} \cdot \frac{\mathrm{x}^{2 \mathrm{k}}}{\Gamma\left(\nu+\frac{1}{2}+\mathrm{k}\right)} \\
& =(\alpha x)^{1 / 2-\nu} \cdot \mathrm{J}_{v-1 / 2}(2 \alpha \mathrm{x})
\end{aligned}
$$

Also, for $v+\frac{1}{2}>0$,

$$
\begin{aligned}
\frac{1}{\Gamma\left(\nu+\frac{1}{2}-\frac{1}{2} \theta\right)}\left(1+\frac{x^{2}}{a}\right)^{-(\nu+1 / 2)} & =\frac{1}{\Gamma\left(\nu+\frac{1}{2}-\frac{1}{2} \theta\right)} \sum_{k=0}^{\infty} \frac{(-1)^{k} \Gamma\left(\nu+\frac{1}{2}+k\right)}{k ! \Gamma\left(\nu+\frac{1}{2}\right)}\left(\frac{x^{2}}{a}\right)^{k} \\
& =\sum_{k=0}^{\infty} \frac{(-1)^{k} \Gamma\left(\nu+\frac{1}{2}+k\right)}{k ! \Gamma\left(\nu+\frac{1}{2}\right)}\left(\frac{1}{a}\right)^{k} \frac{1}{\Gamma\left(\nu+\frac{1}{2}-\frac{1}{2} \theta\right)}\left[x^{2 k}\right] \\
& =\sum_{k=0}^{\infty} \frac{(-1)^{k} \Gamma\left(\nu+\frac{1}{2}+k\right)}{k ! \Gamma\left(\nu+\frac{1}{2}\right)}\left(\frac{1}{a}\right)^{k} \frac{x^{2 k}}{\Gamma\left(\nu+\frac{1}{2}+k\right)} \\
& =\frac{1}{\Gamma\left(\nu+\frac{1}{2}\right)} e^{-\frac{x^{2}}{a}}
\end{aligned}
$$

We shall now consider some properties of the function $G(s, y ; t), s=\sigma+i \tau$, defined as

$$
G(s, y: t)=2^{\nu-3 / 2} \Gamma\left(\nu+\frac{1}{2}\right) t^{-1} e^{-\left(\frac{s^{2}+y^{2}}{4 t}\right)}(s y)^{1 / 2-\nu} I_{\nu-1 / 2}\left(\frac{s y}{2 t}\right),
$$

based on the equation (2.2) above, where $v \geq 0, t>0, y>0$.

LEMMA 2.1. If $G_{V}(s, y ; t)=G(s, y, t)$ is the function defined in the equation (2.7), and $A_{V}$ and $B_{V}$ are some constants, then

$$
\text { (i) }|G(s, y ; t)| \leq\left|A_{\nu} t^{-1 / 2-\nu}\left(\sigma^{2}+\tau^{2}\right)^{-v / 2}\right|_{e}-\frac{(\sigma-y)^{2}-\tau^{2}}{4 t} \text {, }
$$

and 


$$
\text { (ii) } \begin{aligned}
\left|\frac{\partial}{\partial s} G(s, y ; t)\right|= & \left|\frac{s}{2 t}\left[\frac{y^{2}}{2 \nu+1} G_{v+1}(s, y ; t)-G(s, y ; t)\right]\right| \\
\leq & t^{-3 / 2}|y|^{-v}\left(\sigma^{2}+\tau^{2}\right)^{-v / 2} e^{-\frac{(\sigma-y)^{2}-\tau^{2}}{4 t}} \\
& {\left[\left|A_{v} y\right|\left(\sigma^{2}+\tau^{2}\right)^{1 / 2}+\left|B_{v}\right|\right] . }
\end{aligned}
$$

PROOF. By using the asymptotic expansion of the Bessel function $I(z) \sim \frac{e^{z}}{(2 \pi z)^{1 / 2}},|z| \rightarrow \infty$ and definition (2.7) conclusion (i) follows immediately. Conclusion (ii) follows by direct differentiation and making use of conclusion (i).

As direct consequences of the above lemma, we have that

$$
\begin{aligned}
& \text { (i) }|G(x, y ; t)| \leq\left|A_{v}\right| t^{-1 / 2}(x y)^{-v} e^{-\frac{(x-y)^{2}}{4 t}}, \\
& \text { (ii) } \frac{\partial}{\partial s} G(s, y ; t) \text { is a continuous function of the variables } s \text { and } y \text {. }
\end{aligned}
$$

LEMMA 2.2. Let $\int_{0}^{\infty} y^{\nu} e^{-\alpha y^{2}}|\Phi(y)| d y<\infty$, for positive $\alpha$ and $\nu \geq 0$.

Then

$$
U(x, t)=\int_{0}^{\infty} G(x, y ; t) \Phi(y) d \mu(y)
$$

exists for $0 \leq \mathrm{x}<\infty$ and can be analytically extended into the complex plane so that $\mathrm{U}(\mathrm{s}, \mathrm{t})$ is analytic for $\sigma=\operatorname{Re}(s) \geq 0$.

PROOF. Using the estimate (2.10) and the value

$$
d \mu(y)=\frac{2^{1 / 2-v}}{\Gamma\left(\nu+\frac{1}{2}\right)} y^{2 v} d y
$$

we have

$$
\begin{aligned}
|U(x, t)| & \leq \int_{0}^{\infty}|G(x, y ; t) \phi(y) d \mu(y)| \\
& \leq\left|A_{V}(x, t)\right| \int_{0}^{\infty} y^{\nu} e^{-\frac{(x-y)^{2}}{4 t}}|\phi(y)| d y
\end{aligned}
$$

Since

$$
(x-y)^{2} \geq \frac{1}{2} y^{2}-x^{2}, \quad 0 \leq y<\infty
$$


therefore,

$$
e^{-\frac{(x-y)^{2}}{4 t}} \leq e^{\frac{x^{2}}{4 t}-\frac{y^{2}}{8 t}}
$$

and

$$
|U(x, t)| \leq\left|B_{V}(x, t)\right| \int_{0}^{\infty} y^{\nu} e^{-\frac{y^{2}}{8 t}}|\phi(y)| d y<\infty
$$

due to the hypothesis with $\alpha=\frac{1}{8 t}, t>0$. Hence, the integral defining the function $U(x, t)$ exists and is, in fact, absolutely convergent. Now we consider

$$
U(s, t)=\int_{0}^{\infty} G(s, y ; t) \phi(y) d \mu(y), \quad s=\sigma+i \tau \text {. }
$$

Now using the estimate (2.8) of $G(s, y: t)$, we have

$$
\begin{aligned}
|U(s, t)| & \leq \int_{0}^{\infty}|G(s, y ; t) \phi(y) d \mu(y)| \\
& \leq A_{v}(\sigma, \tau, t) \int_{0}^{\infty} y^{\nu} e^{-\frac{(\sigma-y)^{2}}{4 t}}|\phi(y)| d y \\
& \leq A_{v}(\sigma, \tau, t) \int_{0}^{\infty} y^{\nu} e^{-\frac{y^{2}}{8 t}}|\phi(y)| d y<\infty .
\end{aligned}
$$

using the inequality (2.11) and the hypothesis. Hence, the function $U(s, t)$ exists and is defined by an absolutely convergent integral. Now to prove that $U(s, t)$ is analytic in the half-plane $\sigma \geq 0$, we need to show that

$$
\int_{0}^{\infty} \frac{\partial}{\partial s} G(s, y ; t) \phi(y) d \mu(y)
$$

converges uniformly in the region $\sigma \geq 0$.

By making use of the estimate (2.9), we obtain

$$
\begin{aligned}
&\left|\int_{0}^{\infty} \frac{\partial}{\partial s} G(s, y: t) \phi(y) d \mu(y)\right| \leq\left|A_{v}(\sigma, \tau, t)\right| \int_{0}^{\infty} y^{v+1} e^{-\frac{(\sigma-y)^{2}}{4 t}}|\phi(y)| d y \\
&+\left|B_{v}(\sigma, \tau, t)\right| \int_{0}^{\infty} y^{\nu} e^{-\frac{(\sigma-y)^{2}}{4 t}}|\phi(y)| d y
\end{aligned}
$$


Now due to the hypothesis and using the inequality (2.11), both the integrals on the right hand side above, converge for all $s$ and for $t>0$, giving us the desired result and hence the lemma.

As corollaries of Lemma 2.2, we have

$$
|U(s, t)| \leq A_{v}(t)\left(\sigma^{2}+\tau^{2}\right)^{-v / 2} e^{\frac{\sigma^{2}+\tau^{2}}{4 t}}
$$

where $s=\sigma+i \tau$ and $t>0$; and

$$
\begin{aligned}
U(i x, t) & =0\left(e^{\frac{x^{2}}{4 t}}\right), & & x \rightarrow \infty \\
& =0(1), & & x \rightarrow 0 .
\end{aligned}
$$

\section{THE INVERSION.}

We give below a lemma which is a direct consequence of a general result,

[2; Theorem 2.1].

LEMMA 3.1. If $f(x)=2 \int_{0}^{\infty} \phi(t) \frac{1}{t}\left(\frac{t}{x}\right)^{2 v+1} e^{-t^{2} / x^{2}} d t, x>0, v>0$, then

$$
\frac{1}{\Gamma\left(\nu+\frac{1}{2}-\frac{1}{2} \theta\right)}[f(x)]=\phi(x), \quad 0<x<\infty .
$$

PROOF. We write the above integral as

$$
f(x)=\int_{0}^{\infty} \phi(t) \frac{1}{t} k\left(\frac{x}{t}\right) d t
$$

where

$$
k(x)=2 e^{-1 / x^{2}} x^{-(2 \nu+1)}
$$

Now the Mellin transform of $k(x)$ is $k *(s)=\Gamma\left(\nu+\frac{1}{2}-\frac{1}{2} s\right), \sigma<2 \nu+1$ and $\frac{1}{k^{\star}(s)}$ is of Laguerre-Pólya class. Thus

$$
\frac{1}{k^{*}(\theta)} \mathbf{f}(x)=\phi(x) \text { or } \frac{1}{\Gamma\left(\nu+\frac{1}{2}-\frac{1}{2}\right)} f(x)=\phi(x), \quad x>0 .
$$

The Main Theorem: Let $\int_{0}^{\infty}\left|y^{\nu} e^{-\alpha y^{2}} \phi(y)\right| d y<\infty, v>0, \alpha>0$ and

$$
U(x, t)=\int_{0}^{\infty} G(x, y: t) \phi(y) d \mu(y)
$$

be the Poisson-Hankel transform. If 


$$
R(x ; t)=\Gamma\left(\nu+\frac{1}{2}\right) \int_{0}^{\infty} e^{-\frac{v^{2} x^{2}}{16 t^{2}}} G(v ; t) U(i v, t) d \mu(v)
$$

then

$$
\frac{1}{\Gamma\left(\nu+\frac{1}{2}-\frac{1}{2} \phi\right)} \mathrm{R}(\mathrm{x} ; \mathrm{t})=\mathrm{e}^{-\mathrm{x}^{2} / 4 t} \phi(\mathrm{x}), \quad \mathrm{x}>0, \mathrm{t}>0,
$$

where the functions $G(v ; t), G(x, y ; t)$ and $d \mu(v)$ are defined above.

PROOF. From the result (2.8) and the definitions of the functions $G(v: t)$ and $d \mu(v)$, it is clear that the integral defining $R(x ; t)$ exists. Also note that

$$
U(i v, t)=\int_{0}^{\infty} G(i v, y ; t) \phi(y) d \mu(y)
$$

exists due to Lemma 2.2 .

Then we can write

$$
\begin{aligned}
R(x, t) & =\Gamma\left(\nu+\frac{1}{2}\right) \int_{0}^{\infty} e^{-\frac{v^{2} x^{2}}{16 t^{2}}} G(v ; t) d \mu(v) \int_{0}^{\infty} G(i v, y ; t) \phi(y) d \mu(y) \\
& =\Gamma\left(v+\frac{1}{2}\right) \int_{0}^{\infty} \phi(y) d \mu(y) \int_{0}^{\infty} e^{-\frac{v^{2} x^{2}}{16 t^{2}}} G(v ; t) G(i v, y ; t) d \mu(v),
\end{aligned}
$$

the change of order of integration can be justified by absolute convergence; we need only to observe that

$$
\begin{aligned}
& \int_{0}^{\infty}\left|e^{-\frac{v^{2} x^{2}}{16 t^{2}}} G(v ; t) d \mu(v)\right| \int_{0}^{\infty}|G(i v, y ; t) \phi(y) d \mu(y)| \\
& \leq K t^{-(\nu+1)} \int_{0}^{\infty}\left|e^{-\frac{v^{2} x^{2}}{16 t^{2}}} v^{\nu} d v\right| \int_{0}^{\infty} y^{\nu} e^{-y^{2} / 4 t} \phi(y) d y \mid<\infty,
\end{aligned}
$$

by hypothesis.

From the definitions of the functions $G(v ; t), G(i v, y ; t)$ and $d \mu(v)$, the v-integral in (3.2) can be written as 


$$
\begin{gathered}
(2 t)^{-(\nu+3 / 2)} e^{-y^{2} / 4 t} y^{1 / 2-v} \int_{0}^{\omega \nu} e^{-\frac{v^{2} x^{2}}{16 t^{2}}} v^{v+1 / 2} J_{v-1 / 2}\left(\frac{v y}{2 t}\right) d v \\
=2^{v+1 / 2} e^{-y^{2} / 4 t-y^{2} / x^{2}} x^{-(2 v+1)}
\end{gathered}
$$

and we then obtain,

$$
\begin{aligned}
R(x, t) & =2 x^{-(2 v+1)} \int_{0}^{\infty} e^{-y^{2} / 4 t-y^{2} / x^{2} y^{2 \nu}} \phi(y) d y \\
& =2 \int_{0}^{\infty} e^{-y^{2} / 4 t} \phi(y) e^{-y^{2} / x^{2}} \frac{1}{y}\left(\frac{y}{x}\right)^{2 v+1} d y .
\end{aligned}
$$

Now the Lemma (3.1) is applicable and hence

$$
\frac{1}{\Gamma\left(\nu+\frac{1}{2}-\frac{1}{2} \theta\right)} R(x, t)=e^{-x^{2} / 4 t} \phi(x),
$$

establishing the inversion of the Poisson-Hankel transform.

It is to be noted that the transforming function $R(x, t)$ defined by (3.1) is in fact the modified Laplace transform of $U(i x, t)$. This can be recognized if we simplify and write

$$
R(x, t)=(4 t)^{-(\nu+1 / 2)} \int_{0}^{\infty} e^{-p \gamma(x, t)} p^{\nu-1 / 2} U\left(2 p^{1 / 2}, t\right) d p
$$

where $\gamma(x, t)=\frac{x^{2}}{16 t^{2}}+\frac{1}{4 t}, t>0$. Also note that the above inversion algorithm is valid for the entire function $\phi$ having a series expansion. The condition on $\phi$ simply restricts its growth.

Next we shall discuss some special cases. Let $\lim U(x, t)=f(x)$. Then the Poisson-Hankel transform (2.3) becomes the Weierstrass-Hankel transform and is given by

$$
f(x)=\int_{0}^{\infty} G(x, y ; 1) \phi(y) d \mu(y)
$$

Now write $R(x, 1)=R(x)$, so from (3.1)

$$
R(x)=\Gamma\left(v+\frac{1}{2}\right) \int_{0}^{\infty} e^{-\frac{v^{2} x^{2}}{16}} G(v ; 1) f(i v) d \mu(v)
$$

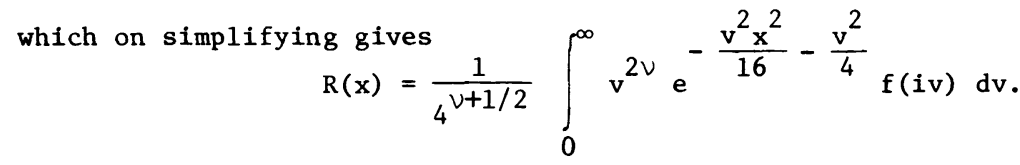


According to the inversion algorithm (3.3), we have

$$
\begin{aligned}
e^{-x^{2} / 4} \phi(x) & =\frac{1}{\Gamma\left(\nu+\frac{1}{2}-\frac{1}{2} \theta\right)} R(x) \\
& =\frac{1}{\Gamma\left(\nu+\frac{1}{2}-\frac{1}{2} \theta\right)} \cdot \frac{1}{4^{\nu+1 / 2}} \int_{0}^{\infty} v^{2 \nu} e^{-\frac{v^{2} x^{2}}{16}-\frac{v^{2}}{4}} f(i v) d v \\
& =\frac{1}{4} \int_{0}^{\nu+1 / 2} v^{2 \nu} e^{-v^{2} / 4} f(i v) d v \cdot \frac{1}{\Gamma\left(\nu+\frac{1}{2}-\frac{1}{2} \theta\right)} e^{-\frac{v^{2} x^{2}}{16}},
\end{aligned}
$$

formally. Now using the result (2.5), we obtain,

$$
e^{-x^{2} / 4} \phi(x)=\frac{1}{4^{v}} \int_{0}^{\infty} e^{-v^{2} / 4} v^{v+1 / 2} x^{1 / 2-v} J_{v-1 / 2}\left(\frac{v x}{2}\right) f(i v) d v .
$$

Thus,

$$
\Phi(x)=\int_{0}^{\infty} G(i x, v ; 1) f(i v) d \mu(v)
$$

giving the inversion of the Weierstrass-Hankel transform and agreeing with the inversion given in [4].

Now if we write $G(0, y ; t)=G(y ; t)$ and $U(0, t)=f(t)$, then the Poisson-Hankel transform given by (2.3) becomes

$$
f(t)=\int_{0}^{\infty} G(y ; t) \phi(y) d \mu(y),
$$

and is called the reduced Poisson-Hankel transform. We can write $1 t$, using the definitions of $\mathrm{G}$ and $\mathrm{d} \mu$ and making a suitable change of variable, as

$$
f\left(t^{2} / 4\right)=\frac{2}{\Gamma\left(\nu+\frac{1}{2}\right)} \int_{0}^{\infty} e^{-y^{2} / t^{2}} \frac{1}{y}\left(\frac{t}{y}\right)^{-(2 v+1)} \phi(y) d y .
$$

Hence by Lemma (3.1), we have

or,

$$
\frac{1}{\Gamma\left(\nu+\frac{1}{2}-\frac{1}{2} \theta\right)} f\left(\frac{x^{2}}{4}\right)=\frac{1}{\Gamma\left(\nu+\frac{1}{2}\right)} \phi(x)
$$

$$
\frac{1}{\Gamma\left(\nu+\frac{1}{2}-\frac{1}{2} \theta\right)} U\left(0, \frac{x^{2}}{4}\right)=\frac{1}{\Gamma\left(\nu+\frac{1}{2}\right)} U(x, 0),
$$


This establishes the inversion of the reduced Poisson-Hankel transform as in [1].

Next we shall illustrate the inversion procedure for the Poisson-Hankel transform by an example.

Let

$$
\phi(x)=x^{\alpha}, 2 v+\alpha>-1, v>-\frac{1}{2}
$$

The function satisfies the condition of the main theorem, and

$$
\begin{aligned}
U(i x, t) & =\int_{0}^{\infty} G(i x, y ; t) y^{\alpha} d u(y) \\
& =(2 t)^{-1} e^{x^{2} / 4 t} x^{1 / 2-\nu} \int_{0}^{\infty} e^{-y^{2} / 4 t} y^{\alpha+v+1 / 2} J_{\nu-1 / 2}\left(\frac{x y}{2 t}\right) d y, \\
& =\frac{\Gamma\left(\nu+\frac{1}{2} \alpha+\frac{1}{2}\right)}{\Gamma\left(\nu+\frac{1}{2}\right)}(4 t)^{1 / 2(\alpha+v+1 / 2)} x^{-1 / 2-\nu} e^{2 / 8 t} M_{1 / 2(\alpha+v+1 / 2), 1 / 2(\nu-1 / 2)}\left(x^{2 / 4 t),}\right.
\end{aligned}
$$

[5, p. 185], M being the Whittaker function.

Now

$$
\begin{aligned}
& R(x, t)=\Gamma\left(\nu+\frac{1}{2}\right) \int_{0}^{\infty} e^{-v^{2} x^{2} / 16 t^{2}} G(v ; t) U(i v: t) d u(v) \\
& =\frac{\Gamma\left(\nu+\frac{1}{2} \alpha+\frac{1}{2}\right)}{\Gamma\left(\nu+\frac{1}{2}\right)}(4 t)^{1 / 2(\alpha-\nu-1 / 2)} \int_{0}^{\infty} e^{-v^{2}\left(\frac{x^{2}}{16 t^{2}}+\frac{1}{8 t}\right)} \\
& v^{v-1 / 2} M_{1 / 2(\alpha+v+1 / 2), 1 / 2(v-1 / 2)}\left(v^{2} / 4 t\right) d v \\
& =\Gamma\left(\nu+\frac{1}{2} \alpha+\frac{1}{2}\right) x^{\alpha}\left(1+\frac{x^{2}}{4 t}\right)^{-(\nu+\alpha / 2+1 / 2)}
\end{aligned}
$$

$[5$, p. 215]

Hence,

$$
\begin{aligned}
\frac{1}{\Gamma\left(\nu+\frac{1}{2}-\frac{1}{2} \theta\right)}[R(x, t)] & =\Gamma\left(\nu+\frac{1}{2} \alpha+\frac{1}{2}\right) \frac{1}{\Gamma\left(\nu+\frac{1}{2}-\frac{1}{2} \theta\right)}\left|x^{\alpha}\left(1+\frac{x^{2}}{4 t}\right)^{-(\nu+\alpha / 2+1 / 2)}\right| \\
& =e^{-x^{2} / 4 t} x^{\alpha} \text { by }(2.3) \\
& =e^{-x^{2} / 4 t} \phi(x)
\end{aligned}
$$


according to the main theorem, whence, as predicted,

$$
\phi(x)=x^{\alpha} .
$$

\section{REFERENCES}

1. HAIMO, D.T. and CHOLEWINSKI, F.M. Inversion of the reduced Poisson-Hanke1 Transform, J. d'Analyse Math. 25 (1972), 323-343.

2. WIDDER, D.V. Inversion of a Convolution Transform by use of Series, J. d'Analyse Math. 21 (1968), 293-312.

3. NASIM, C. An Inversion Formula for Hankel Transform, Pacific J. of Math., Vo1. 57, No. 1, 1975.

4. CHOLEWINSKI, F.M: and HAIMO, D.T. The Weierstrass Hankel Convolution Transform, J. d'Analyse Math. 17 (1966), 1-58.

5. ERDELYI, A. et al. Tables of Integral Transform, Vol. 2, New York, 1954. 


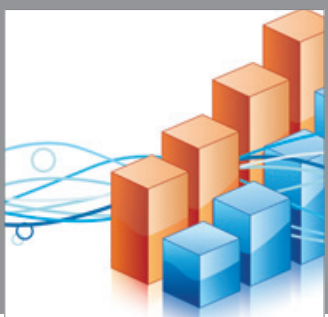

Advances in

Operations Research

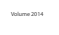

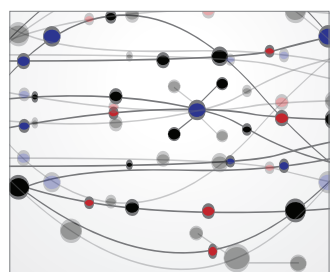

\section{The Scientific} World Journal
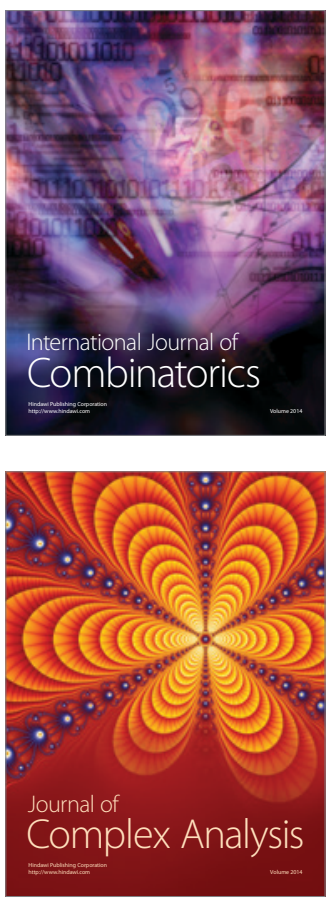

International Journal of

Mathematics and

Mathematical

Sciences
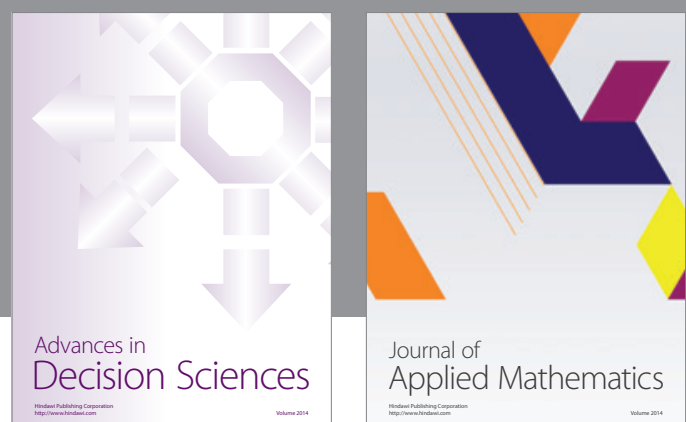

Journal of

Applied Mathematics
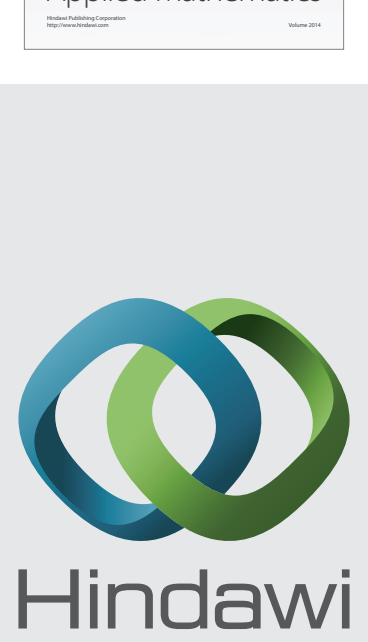

Submit your manuscripts at http://www.hindawi.com
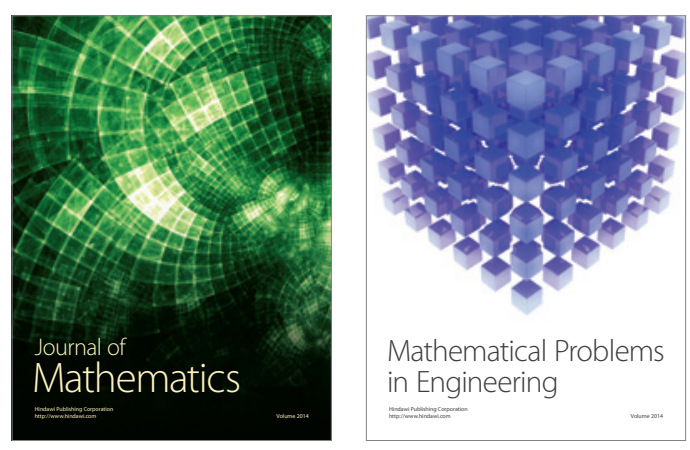

Mathematical Problems in Engineering
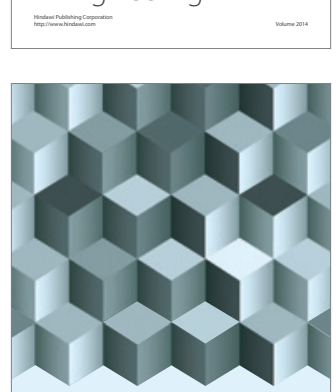

Journal of

Function Spaces
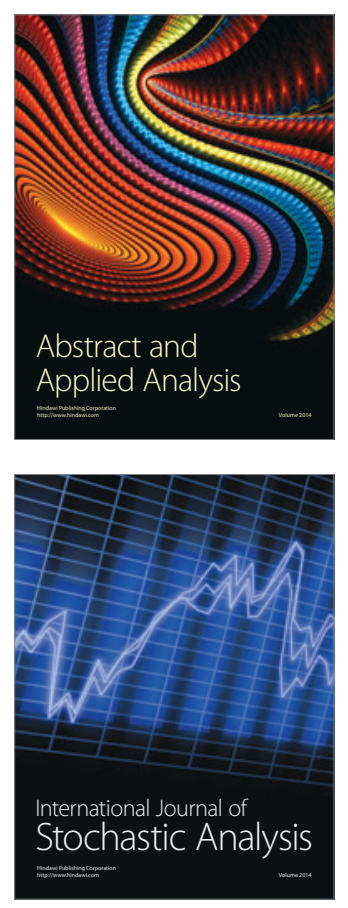

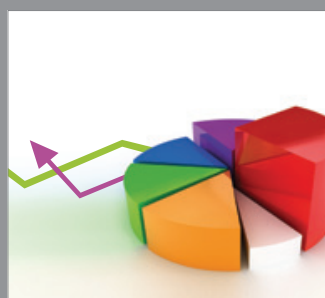

ournal of

Probability and Statistics

Promensencen
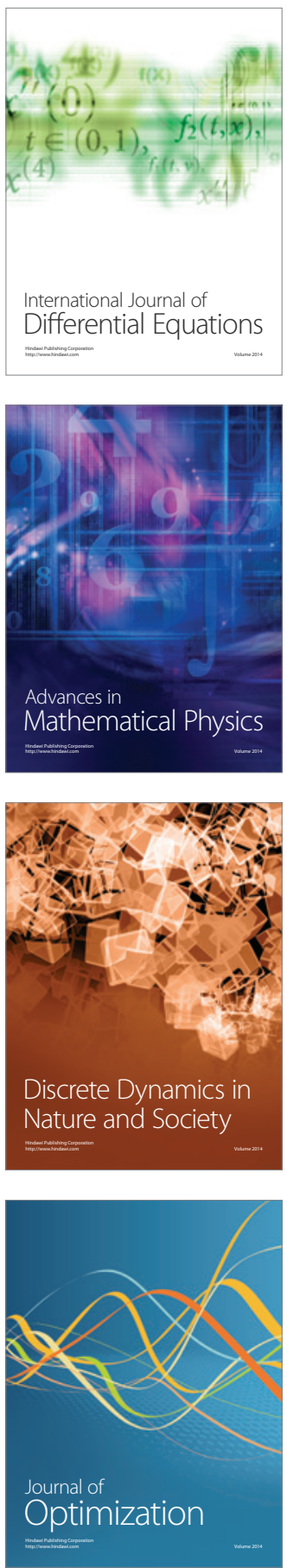